\title{
El cuerpo en Florence Nightingale: un diálogo con la fenomenología existencial de Merleau-Ponty
}

\author{
Florence Nightingale's body concept: a dialogue with Merleau-Ponty's existential phenomenology \\ O corpo em Florence Nightingale: um diálogo com a fenomenologia existencial de Merleau-Ponty
}

\section{Sinthya Allison Schubert Calderón ${ }^{1}$ [D \\ Benjamín Félix Vera Becerra ${ }^{1}$ (1) \\ Edith Elina Rivas Riveros ${ }^{1}$ (D)}

1. Universidad de La Frontera. Temuco, Chile.

\begin{abstract}
Resumen
Objetivos: El propósito es develar el concepto de cuerpo nightingaleano al compararlo con la fenomenología corporal de Merleau-Ponty. Método: Análisis hermenéutico-cualitativo del texto "Notas sobre Enfermería: qué es y qué no es", en conjunto con Fenomenología de la Percepción. Dado el enfrentamiento a un texto propiamente, el instrumental de interpretación se ha sostenido sobre la hermenéutica filosófica propuesta por Hans-Georg Gadamer. Resultados: Florence, en Notas sobre Enfermería propone rudimentariamente un sistema comprensivo del cuerpo como eje del aparecer de la realidad, donde el quehacer enfermero es comprender cómo este aparecer del paciente configura la percepción de su mundo y coopera en unirlo a una nueva situación vital que exige una percepción de ánimo frente a su propia recuperación, nacido del diálogo con MerleauPonty. Conclusiones e implicaciones para la práctica: La comprensión de lo que se interviene es primordial para el ejercicio profesional enfermero. Desde Nightingale, entonces, lo esencial para la disciplina debe ser entender el aparecer de la realidad del paciente para de intervenir en él en su totalidad.
\end{abstract}

Palabras claves: Cuerpo humano; Existencialismo; Filosofía en Enfermería; Hermenéutica; Conocimiento.

\section{Abstract}

Objectives: The purpose is to develop Nightingale's body concept by comparing it with the Merleau-Ponty's existential phenomenology. Method:This is an hermeneutic-qualitative analysis of the text Notes on Nursing: What is and what is not, together with Phenomenology of Perception. Given the confrontation with a text, the instrumental of interpretation has been sustained on the philosophical hermeneutics proposed by Hans-Georg Gadamer. Results: Florence in Notes on Nursing rudimentarily proposes a comprehensive system of the body as the axis of appearance of reality, where the nursing task is to understand how this appearance of the patient configures the perception of his world and cooperates in uniting him to a new vital situation that requires a perception of spirit in the face of his own recovery, born from dialogue with Merleau-Ponty. Conclusion and implication for practice: Understanding what is involved is essential for the professional nursing practice. Since Nightingale then, what is essential for discipline must be understood as the appearance of the reality of the patient to intervene in him as a whole.

Keywords: Human Body; Existentialism; Nursing Philosophy; Hermeneutics; Knowledge.

\section{Resumo}

Objetivos: O propósito é develar o conceito de corpo em Nightingale ao compará-lo com a fenomenologia existencial de MerleauPonty. Método: Análise qualitativo-hermenêutico do texto Notas sobre Enfermagem: o que é e o que não é, com Fenomenologia da Percepção. Dado o confronto com o texto, o instrumento de interpretação tem o sustento sobre a hermenêutica filosófica de Hans-Georg Gadamer. Resultados: Florence em Notas sobre Enfermagem propõe um rudimentar sistema compreensivo do corpo como o eixo de aparecer da realidade, onde o trabalho é entender como o aparecer do paciente configura a percepção de seu mundo e colabora em uni-lo com uma nova situação vital que exige uma percepção de encorajamento frente a sua própria recuperação, revelado após dialogar com Merleau-Ponty. Conclusões e implicações para a prática: A compreensão do que se intervem é primordial para o exercício profissional da enfermagem. Então, desde Nightingale o essencial para a disciplina deve ser a compreensão do aparecer da realidade do paciente para intervir nele, na sua totalidade.

Palavras-chave: Corpo Humano; Existencialismo; Filosofia em Enfermagem; Hermenêutica; Conhecimento.
Autor correspondiente

Sinthya Allison Schubert Calderón.

E-mail: sinthya.schubert@ufrontera.cl

Recibido en 01/07/2020.

Aprobado en 02/09/2020. 


\section{INTRODUCCIÓN}

La tradición histórica de la herencia cartesiana ha comprendido al cuerpo humano como un objeto medible sujeto a las leyes del mundo físico. Tras el giro copernicano de la filosofía kantiana, emergen comprensiones de cuerpo en otras escuelas de pensamiento, entre ellas la fenomenología, que lo viene a comprender como posibilidad de acceso al mundo y encarnación de este, puntualmente en la tradición existencial ${ }^{1}$. Análogamente se interpreta al cuerpo como un objeto de estudio molecular y sus interacciones inter-sistemas permiten su definición ${ }^{2}$ derivado de la comprensión cartesiana referida, siendo analizable físicamente; cobrando relevancia la pregunta por su naturaleza y siendo respondida por autores que proponen distintas comprensiones del mismo $0^{1,3-5}$.

En aproximación al cuerpo, la disciplina enfermera se ha propuesto dilucidar los conceptos salud, enfermedad, persona y enfermería, en cada modelo o teoría ${ }^{6}$ con el fin de definir sus prácticas y con ello permitir en el paradigma un mecanismo de intervención 7 . De ahí el cuestionamiento actual acerca de las limitaciones teóricas de las mismas se constituya una plataforma de desarrollo de la disciplina enfermera abriéndose a otras áreas del conocimiento.

Florence Nightingale, iniciadora del movimiento enfermero moderno, desarrolla una inquietud disciplinar motivada por una formación en ideales sociales y desarrollos teóricos matemáticos y filosóficos ${ }^{8-11}$, sumado a un contexto salubrista de alto riesgo, donde las mayores determinantes de salud del momento eran la situación socioeconómica por un lado y las condiciones de vida por otro, evidenciado por la esperanza de vida que para esa época era de 49 años en la clase profesional, 27 años en comerciantes, y 16 años en obreros. Las ciudades victorianas tenían escasez de alcantarillado, mataderos sin regulación y calles sin saneamiento ${ }^{12,13}$. En este contexto estalla la guerra de Crimea, y frente a la presencia de condiciones inadecuadas en los hospitales militares de aquel tiempo ${ }^{14}$, se solicita a Nightingale que organice una expedición de enfermeras para mejorar la condiciones sanitarias, interviniendo efectivamente ${ }^{13}$ hasta disminuir la mortalidad del 60 al $2.2 \%{ }^{8}$.

Florence es la primera en generar conocimiento enfermero al redactar posterior a su experiencia en la guerra "Notes on Nursing: What is and What is Not" expresando su motivación hacia su profesionalización a través de la educación ${ }^{10}$, debido a que en el siglo XIX las mujeres que ejecutaban el cuidado de los enfermos no tenían educación formal, y desempeñaban esta función en hogares y establecimientos de salud ${ }^{8,10,12}$. Bajo su pensamiento explica el significado de los fenómenos observados a través del análisis, el razonamiento y la argumentación lógica ${ }^{6}$, relacionándolos con su orientación filosófica sobre la interacción paciente-entorno ${ }^{9,10}$, definiendo así la acción del cuidar ${ }^{5}$. En este libro Nightingale problematiza entre muchos conceptos, la corporalidad, describiendo la situación de enfermar como única y subrayando su vivencia.

Hasta nuestros días se describe en el ejercicio enfermero prácticas de cuidado influenciadas por Florence, tales como interpretaciones del actuar de enfermeras(os) en la interacción con los otros y actos de cuidado ${ }^{14}$, situaciones de responsabilidad profesional y roles que se representan con las interacciones corporales en el escenario del cuidado de la salud ${ }^{15}$.

Por su parte, Maurice Merleau-Ponty, discute la corporalidad entendiéndola desde el existencialismo, paradigma del cuerpo subjetivo, esto es, el cuerpo es la forma de percepción de la realidad, donde este es quien encarna la situación que vive $e^{3,16}$.

El presente trabajo pretende abordar la interpretación de cuerpo/persona en Notas sobre Enfermería: Qué es y Qué no es de Florence Nightingale, desde la perspectiva hermenéutica de Gadamer, en contraste con la fenomenología existencial Merleaupontina en Fenomenología de la Percepción.

\section{MÉTODO}

Se realiza un análisis hermenéutico-cualitativo de los textos Notas sobre Enfermería: Qué es y Qué no es, en conjunto con Fenomenología de la Percepción. Dado el enfrentamiento a los textos, el instrumental de interpretación se ha sostenido sobre la hermenéutica filosófica propuesta por Gadamer ${ }^{17}$. Para este pensador, el acceso al texto se da en el sentido existencial del encuentro con un otro ${ }^{18,19}$. En este paradigma, la comprensión acontece en situación dialogante sostenido entre el lector y el texto, sin imposiciones del que comprende al escrito ${ }^{20}$, sino que en una actitud de apertura ocurrida en la interpelación mutua, sucede la suprema de la hermenéutica de la disposición del investigador a dejarse decir algo por texto con el que se encuentra ${ }^{18}$.

Koskinen ${ }^{21}$, en su artículo "Hermeneutic reading of classic texts" identifica un modelo de interpretación de textos para la investigación en las ciencias del cuidado, tomando como referencia al autor mencionado.

Este modelo comienza con (i) una primera lectura del texto elegido en su unicidad reconociéndola como un otro; luego (ii) los párrafos que tocaron y asombraron al lector se extraen del texto; posteriormente (iii) una nueva lectura cuidadosa y flexible de los fragmentos extraídos se realiza, hallando patrones de significado que tengan relevancia para el cuidado; y finalmente (iv) estos patrones son interpretados para una nueva comprensión de fenómenos de la ciencia del cuidado.

La lectura hermenéutica es un trabajo individual que propone al investigador como lector que adopta una actitud de apertura hacia el texto que le permite su comprensión, encontrando patrones significantes que guardan relación con la base teórica del cuidado ${ }^{20}$.

El proceso de interpretación de estos libros clásicos se llevó a cabo a través de la lectura hermenéutica, triangulando la información mediante dos lectores para la validez interpretativa.

Los estadios de la lectura fueron: (i) Una lectura completa del libro de Nightingale permitiendo que hable por sí mismo en su unicidad temporal. (ii) Hubo extractos de los capítulos abordados sobre el cuerpo (descritos en el Prólogo, en los capítulos Variedad, Charlatenería que da esperanza y consejos y Observación del enfermo $)^{5}$ que se presentaron en curiosidad y asombro. (iii) Se realizó una segunda lectura rigurosa de 
los mismos, además de ser discutidos por los investigadores en cuanto a su interpretación, abriéndose estos fragmentos desde la Fenomenología de la Percepción de Merleau-Ponty. (iv) El concepto de cuerpo/ser paciente entendido por el dogma disciplinar enfermero es superado por Florence, ya que sugiere una nueva comprensión del mismo, incluso reinterpretando el modelo teórico ambientalista donde ha sido clasificada históricamente.

Respecto del texto Fenomenología de la Percepción, (i) se realiza una lectura del libro permitiendo su aparecer en otredad, como un texto de profundización en la corriente fenomenológica post-husserliana. (ii) Destacan porciones del texto donde aborda la espacialidad del propio cuerpo. (iii) Al releer se perciben coherencias entre lo descrito por Nightingale y por MerleauPonty. (iv) En Merleau-Ponty se consagra al propio cuerpo como mecanismo de apertura de realidad, posibilitadora de la percepción.

Se consideran los criterios éticos de Ezekiel Emanuel, de valor, por la aportación contingente de la crítica a la disciplina y sus conceptualizaciones teóricas, de validez por la consideración de robusta carga teórica, selección del texto según el valor clásico de la obra, considerando al mismo tiempo los beneficios de esta interpretación sobre sus riesgos, sin conflictos de intereses, contemplando que hay respeto y manejo de la información de los textos bajo el debido reconocimiento del trabajo de sus autores.

\section{RESULTADOS Y DISCUSIÓN}

\section{Corporalidad en Merleau-Ponty: Cuerpo Vivido.}

La filosofía de Merleau-Ponty en "Fenomenología de la Percepción", tiene en cuenta el valor del yo como subjetividad en el proceso perceptivo, no como una relatividad pero universal: ¿Es acaso que las cosas tienen en sí mismas un espacio, o bien, un 'estar en' si no de hecho, siempre referidas a un alguien que las interprete como 'estando en'?

La pregunta acerca de qué es el cuerpo cobra sentido en un contexto de auge racionalista de la época; desde la fenomenología (disciplina filosófica de Edmund Husserl) la cuestión del problema perceptual emerge como dilema de la intencionalidad (las cosas aparecen siempre referidas de algo, hacia sí), de ahí que Merleau-Ponty constituya la percepción del cuerpo como intencionalidad (esto es, la percepción de las cosas se aparecen en referencia a la corporalidad) ${ }^{22}$. MerleauPonty dirá: Si tengo mi brazo encima de la mesa, nunca se me ocurrirá decir que está al lado del cenicero como éste lo está del teléfono. El contorno de mi cuerpo es una frontera que las relaciones ordinarias de espacio no franquean. ${ }^{3: 115}$

La corporalidad se vive, y no se comprende en un espacio físico, en tal caso entendido como un cuerpo encapsulado "aquí" lleno de órganos actuando en la realidad, dado hacia uno distinto del sí mismo; sino en un espacio vivido, o bien, encarnado de la situación en la que el cuerpo está tanto ahí como acá; es decir, la corporalidad es espacio que abre a la realidad, o sea, corporalidad es un acá en cuanto "yo", y un ahí en cuanto "yo", como aperturas a la realidad (en adelante, mundo).
Las palabras que permiten explicar el fenómeno de un cuerpo ahí y acá pueden relacionarse con las originales del alemán: Leib y Körper, entendiendo a Leib como vida o un cuerpo vivido, y a Körper como cuerpo orgánico, o físico. La relación con el "ahí" y el "acá" serán el desarrollo del pensamiento Merleau-pontino, aunque a primera vista se relacione el "acá" con el cuerpo orgánico (Körper), y el Leib como su gran tesis; un cuerpo que es un "acá" pero que es vivido, y es vivido también en un "allá", es decir, un cuerpo Körper-Leib desde el cual el mundo se abre ${ }^{3}$.

En cuanto a ser el cuerpo, Merleau-Ponty refiere al ejemplificar el reconocimiento del cuerpo propio: Nosotros no contemplamos únicamente las relaciones de los segmentos de nuestro cuerpo y las correlaciones del cuerpo visual y del cuerpo táctil: somos nosotros mismos el que mantiene juntos esos brazos y piernas... ${ }^{3: 167}$

Se infiere que el cuerpo se interpreta a sí en su percepción. Al ver el cuerpo, no se está delante de él como fuera, sino siendo él (no se tiene un cuerpo, se es un cuerpo) ${ }^{3}$.

Comprendiendo lo mencionado en los párrafos anteriores se desprende que lo esencial en el cuerpo es su poder ser, el cuerpo es posibilidad. Como el elemento que tiene la facultad de ponerse en el mundo, bien entendido desde la tradición hasta este punto, un "yo" que no es en sí, sino en relación con el mundo que se le aparece. Esta relación corporal se traduce en un poder ser quien soy en el mundo de las cosas.

Por ejemplo, para la situación de un paciente, el estar enfermo no es solo la enfermedad que ocurre en una herida y sus procesos moleculares reparativos (el cuerpo "aqui"), sino también ocurre que esa aproximación psíquica a su realidad (es decir, su percepción "fuera de su cuerpo") está "allá". Las paredes podrían parecerle más grises y el mundo más triste por su enfermedad, ${ }^{5}$ pues todo su ser está enfermo; tal situación de "afuera", del gris de la pared y el mundo triste, no es meramente las características ambientales, sino que en su situación de enfermedad, el cuerpo es ese mismo gris de afuera y el aspecto triste, tanto como su herida física.

Lo anterior se hace gráfico al desplegar la corporalidad en el mundo, donde el cuerpo va encarnando las posibilidades de ser. Una frase que lo grafica es: nuestra conciencia cambia de cuerpo 23:106-107, es decir la corporalidad en cuanto relación con el mundo, no es sólo una relación de un "yo acá" con un "yo allá" meramente, uniendo ambas identidades. Para evitar tal cuestión se debe hablar de la encarnación del "yo" en su mundo. El "yo acá" y el "yo ahí", una constitución de totalidad sintética de cuerpo-mundo.

Esto es que, el cuerpo en cuanto Körper es a la vez no sólo una cápsula física sino un Leib, es decir, el cuerpo vivido unido a su situación vital; esta situación vital es el despliegue mismo de su corporalidad vivida. De otro modo, "yo soy" la situación que se vive, "yo encarno" la situación, luego "yo encarno el mundo", y sin extremar, al cambiar mi situación luego cambia con ello el cuerpo. La dimensión Leib afecta la situación vital y con ello el cuerpo, y con el cuerpo, esta totalidad sintética Körper-Leib, de esta forma "la conciencia cambia de cuerpo". Para MerleauPonty, el cuerpo es a la vez vida orgánica y física, pero también 
una expresión del "yo" unido a su situación vital, desplegándose en ella; por ende, cada situación también exige de un cuerpo que la encarne.

\section{Corporalidad en Florence Nightingale}

Cuando se considera a Notas, como un libro del quehacer enfermeril, se describirá experiencialmente cómo es cuidar del enfermo. Florence observa cómo es el enfermar del paciente y no desde la enfermedad como si pudieran separarse ${ }^{5}$. Las nociones del ser cuerpo en Florence bordean fronteras dualistas cristianas por la cosmovisión expresada ${ }^{8}$, pero resaltan elementos que escapan de esta visión tradicional del cuerpo, evidenciando nociones de "ser cuerpo" y "ser un cuerpo ahí" como las siguientes.

\section{Cuerpo como posibilidad de enfermar y sanar}

Florence hace hincapié en la posibilidad natural del cuerpo de enfermar y sanar tratándolo como una organicidad total capaz de regeneración, otorgándole una noción de poder ser restaurador por condiciones innatas, a diferencia de una visión mecanicista en la que se hace uso de herramientas para su restauración biológica.

El proceso reparador que la naturaleza ha instituido, y que nosotros llamamos enfermedad, ha sido entorpecido[...]si un paciente tiene frío, o fiebre, o está mareado [...] no es culpa de la enfermedad, sino de la enfermería [...] (que) se ha limitado a significar poco más que la administración de medicamentos y la aplicación de cataplasmas[...](El arte de la enfermería) parece ser expresamente establecido para deshacer lo que Dios ha hecho que sea la enfermedad, es decir, un proceso reparador:2.

La enfermedad se va constituyendo como proceso del cuerpo mismo de sanidad, por tanto no es una situación mecánica de daño entendida como serie de procesos fisiopatológicos que se describen molecularmente, el enfoque es tratar las condiciones que el cuerpo sabe que posibilitan su salud. De ahí que insista que la enfermería es principalmente observación y cuidado, por sobre la técnica de administración de medicamentos y cataplasmas.

Al tratar la importancia vital de la observación profunda, se debe perder de vista cuál es su fin. No es para amontonar más información sobre hechos curiosos, sino para salvar vidas y aumentar la salud y la comodidad. [...] Es bastante sorprendente ver cómo tantos hombres (también hay mujeres que lo hacen) se comportan prácticamente como si el fin científico fuera el único que se persigue, o como si el cuerpo enfermo no fuera más que un depósito para guardar medicamentos y la enfermedad quirúrgica, sólo un caso curioso que el que la sufre ha originado, para información especial del que lo atiende ${ }^{5: 124}$.

\section{Cuerpo perceptual desde la interioridad.}

Florence reflexiona al cuerpo como posibilidad de aparecer de mundo perceptual, en que la vulnerabilidad es manifestada psíquicamente "afuera": el enfermo es lábil a la angustia, y su mundo puede ser configurado en preocupantes problemas.

Ustedes creen estar sobrecargados de ansiedades, pero que tienen la posibilidad de pasear cada día por la calle Regent o por la campiña, o comer con otros en otras habitaciones, etc., poco saben de cómo sus ansiedades se suavizan por ello, y de lo mucho que se intensifican en aquellos que nunca pueden cambiar: cómo hasta los muros de sus habitaciones de enfermo parecen colgar junto con sus preocupaciones; cómo los fantasmas de sus problemas aparecen en sus camas, y cuán difícil es para ellos escapar a la persecución de un pensamiento sin la ayuda de algún cambio, de alguna variedad 5:57.

Destaca que la configuración de la enfermedad física influye en la estructura perceptiva del mundo del enfermo, y lo contrario también es cierto; la manipulación de esto puede influir en su estado físico, no como una estimulación meramente ambiental, sino desde el reconocer el malestar subjetivamente: Poco es lo que se sabe o comprende del sufrimiento real de la enfermedad. Y qué poca idea tiene nadie de buena salud acerca de la vida de una persona enferma. ${ }^{5: 101}$ Es acompañar a una situación de mejoramiento considerando que su ambiente sea adaptado conforme su bienestar emocional porque afectaría la situación en sí de enfermedad.

\section{Cuerpo sintético de realidad y organicidad}

Desde el mismo apartado anterior, cabe señalar la noción de cuerpo que Florence entiende es un cuerpo total y no una integralidad que se reune. A raíz de esta rudimentaria comprensión sintética (más que una suma de las partes, es una totalidad en sí), se plantea entonces una percepción del mundo desde la interioridad, implicando así un concepto de cuerpo que es más que organismo funcional actor principal de la realidad, sino un engrajane sintético cuyas relaciones entre sí se manifiestan como "influencia del cuerpo sobre el espíritu"y al revés: Se han escrito volúmenes y mucho se habla actualmente acerca de la influencia del espíritu sobre el cuerpo. Mucho de ello es verdad. Pero me gustaría que se pensara un poco más en la influencia del cuerpo sobre el espíritu. Es una cuestión de cruel sorpresa para los propios enfermos ver cómo predominan las ideas dolorosas sobre las agradables en sus impresiones; ellos razonan para sí; se creen ingratos; nada sirve para nada... $5: 56$

Esto es, reconocimiento del "poder ser" no sólo desde la interioridad, pero desde la exterioridad que configura su estado subjetivo también.

El presente escrito logra develar aspectos teóricos desde su historia. Se conceptualiza el cuerpo en Florence en contraste con el pensamiento contemporáneo de Merleau-Ponty, abriendo fructíferamente esta noción. Se describen a modo de conclusión estas similitudes halladas, se discuten y finalmente se reflexionan las implicancias de la comprensión abordada.

El cuerpo en ambos no se constituye como algo que se posee, antes bien como uno que se es, del cual las fronteras perceptuales se configuran como mundo. Esta concepción desde el cuerpo es una conclusión desprendida de la percepción para Merleau-Ponty, como encarnación de la situación, y desde Nightingale como aparición de la interioridad psíquica en el mundo percibido; tanto en uno como en el otro existe una forma de "situaciones que exigen de cuerpo" (Figura 1). 


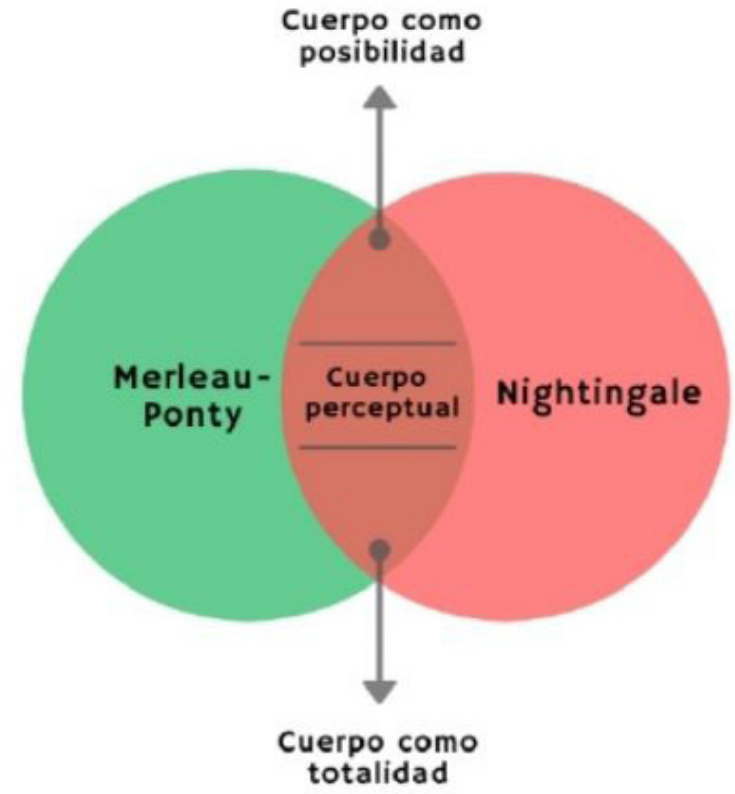

Figura 1. Resumen aspectos comunes en corporalidad entre Maurice Merleau-Ponty y Florence Nightingale.

La concepción de interioridad en Nightingale es homologable al concepto de "cuerpo aquí" en Merleau-Ponty, tanto como de exterioridad (ambiental) a "cuerpo ahí", entendiéndolo en ambos como la unidad que es capaz de sintetizar el mundo en sí, en su dimensión perceptiva de la realidad del cuerpo "biológico" y de su "situación".

Finalmente, destaca la aplicación de una superación dualista (es decir separación de la psiquis o alma del cuerpo material) en Nightingale, a través de la noción corporal de aparecer de mundo desde la interioridad. Lo anterior configura elementos básicos de la noción de ser humano que apuntan no meramente a una visión dimensional del ser (un espíritu, alma y cuerpo, o biopsicosocial), sino más bien a una integración del humano que es totalidad sintética. Siendo envuelta en su situación de enfermedad (de ahí el énfasis en los doce capítulos), puede al mismo tiempo ser restaurado en su materialidad, cambiando desde su interioridad la manifestación de su exterioridad; por tanto se concluye que el aparecer del mundo se vuelve el área más importante de la disciplina enfermera.

Unas rudimentarias ideas de la corporalidad fenomenológica se hallan (Figura 2), quien más que realizar un tratado positivista, desde la más pura experiencia muestra con simpleza la totalidad sintética que es existir a través del cuerpo, especialmente desde el enfermo, vanguardista de una elemental dimensión fenomenológica del ser.

Se ha escrito sobre el pensamiento Nightingaleano ideas que al contrastarlas con estos resultados desde Notas sobre Enfermería, evidencian diferencias de valor.

Según Núñez ${ }^{24}$, a través de Nightingale podemos comprender la historia de la enfermería y su desarrollo teórico al ser ella

\section{Florence Nightingale}

1859

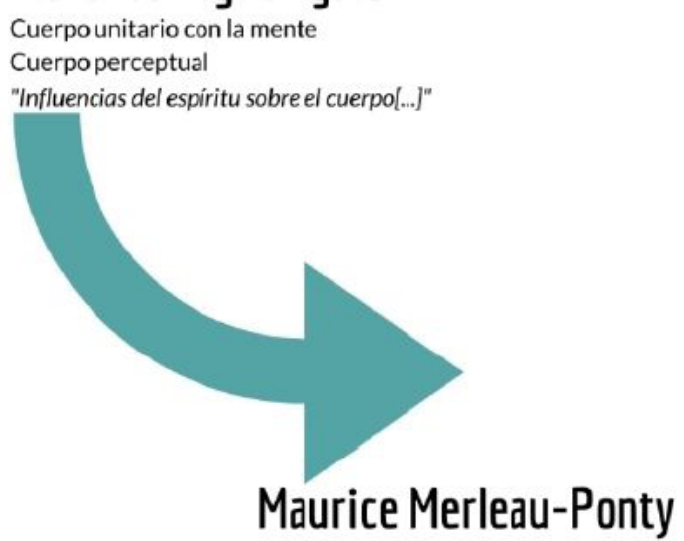

1945

po como totalidad sintética

Cuerpo que es la realidad* misma percibida

Figura 2. Distinciones filosóficas de la corporalidad entre Florence Nightingale (Notas sobre Enfermería) y Maurice Mearleau-Ponty (Fenomenología de la percepción).

pionera en traspasar los conocimientos a un escrito develando los conceptos rudimentarios de la disciplina. Según esta autora, el cúmulo de saberes de la profesión desembocan en el cuidar del ambiente como forma de prevención o recuperación de la salud. También Pfettscher va a asentar en la autora la teoría ambientalista como primer paso hacia los modelos teóricos de enfermería que la fundamentarán como disciplina que reflexiona y que valida su propio quehacer ${ }^{25}$. Se trae entonces a esto la propia reflexión de Nightingale en reiterar no hacer un manual, sino un espacio reflexivo del quehacer enfermero, actividad que para ella misma ${ }^{5}$ no se trata meramente del entorno y su influencia en la recuperación del paciente, sino en un mecanismo comprensivo de lo que llama la influencia del cuerpo sobre el espíritu, que permite el aparecer mismo de las cosas del mundo desde su enfermedad, sobre el cual la profesión no se circunscribe a la sola administración de elementos externos que modifican un ambiente. Otros autores proponen esta misma teoría en Florence, donde históricamente se le ha situado, en la que al modificar el ambiente mejorase per se la calidad de restauración del individuo ${ }^{25-27}$; lo que se propone en esta lectura en cambio, es considerar la comprensión del sujeto en su totalidad ${ }^{28}$, donde la enfermedad es aquello situacional que envuelve el mundo del afectado. De ahí que la intervención propiamente se realice en la comprensión de este aparecer.

Mientras que otra autora propone en Florence un obstáculo epistémico para el desarrollo disciplinar científico de la enfermería ${ }^{29}$; se reconoce en esta lectura una aproximación al desarrollo epistémico contrario a la visión positivista, dado que hay una aproximación a la definición y comprensión de cuerpo mucho más cercana a las discusiones filosóficas que su aproximación como "cosa del mundo", propia del racionalismo y su desarrollo positivista ulterior ${ }^{1,30}$. Al entenderse conceptualmente el cuerpo del 
modo descrito, tiene como consecuencia una deshumanización de las personas en el sistema de salud ${ }^{31}$.

\section{CONCLUSIONES E IMPLICANCIAS PARA LA PRÁCTICA}

Las implicancias que alcanzan las comprensiones mecanicistas del cuerpo en la profesión enfermeray sanitaria tienen consecuencias actuales en su ejercicio. Un enfoque del cuerpo deshumanizado le sujeta a las cosas del mundo. Un ejemplo de ello es que exista una priorización de los indicadores de un centro de salud por sobre el bienestar experiencial de las personas atendidas por él. En esto la comprensión propuesta por Nightingale desde la práctica misma supera el abordaje cuantitativo o mecanicista del sistema, abriendo el camino hacia nuevas preguntas por el sujeto al que se debe la profesión.

Al percibirse un desconocimiento de las problemáticas filosóficas, se observa una apertura que abre un espacio para la alta tecnificación y el olvido del cuerpo representado en una menor orientación teórica de su ser. Abrir la filosofía en la enfermería permitiría pensar y crear una nueva manera de analizar las problemáticas disciplinares que cuestione los valores e ideas existentes, no solamente por actuar según la ciencia en tanto que positiva, sino que con comprensiones integrales relacionadas con las personas y su realidad.

Urge el desarrollo de un pensamiento crítico y reflexivo en la formación y el ejercicio profesional con base en la enfermedad, para así continuamente plantearse interrogantes a responder por la filosofía, necesaria para desarrollar una enfermería centrada en la persona.

En vista de lo interpretado del libro mismo, se vislumbran nuevos horizontes desde los lentes que Florence misma ofrece. El desafío recae entonces en comprender sus escritos, los cuales más que manual de instrucciones para la práctica, son un tratado del comienzo de la experiencia disciplinar. Cabe preguntarse qué intencionó ella al momento de escribir y qué dice hoy en cuanto a conceptos trascendentes para la enfermería. Se considera que su reclamo último a la luz de Notas es totalmente actual: poco se comprende qué es y quién es el objeto de interés para las ciencias del cuidado, al asumir al cuerpo meramente como el sistema orgánico, se pierde entonces su corporalidad, la cual es la fuerza orientadora y fundamental de lo que es y no es enfermería.

\section{CONTRIBUICIONES DE LOS AUTORES}

Concepción del diseño del estudio de revisión. Sinthya Allison Schubert Calderón. Benjamín Félix Vera Becerra.

Aquisición de bibliografía para la revisión. Sinthya Allison Schubert Calderón. Benjamín Félix Vera Becerra.

Análisis de datos e interpretación de resultados. Sinthya Allison Schubert Calderón. Benjamín Félix Vera Becerra. Edith Elina Rivas Riveros
Redacción y revisión crítica del manuscrito. Sinthya Allison Schubert Calderón. Benjamín Félix Vera Becerra. Edith Elina Rivas Riveros

Aprobación de la versión final del artículo. Sinthya Allison Schubert Calderón. Benjamín Félix Vera Becerra. Edith Elina Rivas Riveros

Responsabilidad por todos los aspectos del contenido y la integridad del artículo publicado.

Sinthya Allison Schubert Calderón. Benjamín Félix Vera Becerra. Edith Elina Rivas Riveros

\section{EDITOR ASSOCIADO}

Sofia Sabina Lavado Huarcaya

\section{REFERÊNCIAS}

1. Johnson F. Hacia la pregunta por la coporalidad: reflexiones sobre el cuerpo humano en cuanto organismo. Alpha (Osorno). 2009;29(1):16784.

2. Hall JE. Tratado de fisiología médica. Barcelona: Elsevier; 2011.

3. Merleau-Ponty M. Fenomenología de la percepción. 2a ed. Buenos Aires: Planeta-Agostini; 1993.

4. Leder D. A tale of two bodies: the cartesian corpose and the lived body. Medical thought and practice. Maryland: Kluwer Academic Publishers; 1992. p. 17-35.

5. Nightingale F. Notas sobre Enfermería: qué es y qué no es. Madrid España: Masson; 2002.

6. Alligood M. Introducción a las teorías en enfermería: historia, importancia y análisis. In: Rodríguez M, editor. Modelos y teorías en enfermería. Greenville: Elsevier; 2011. p. 3-15.

7. Young P, Hortis V, Chambi M, Finn B. Florence Nightingale (1820-1919), a 101 años de su fallecimiento. Rev Med Chile. 2011; 139(6):807-13 http://dx.doi.org/10.4067/S0034-98872011000600017.

8. Hanzeliková A, Carabaño MJ, Torresano B, García MR. Florence Nightingale y el contexto socio-cultural de su época. Cultura de los Cuidados. 2005; 18(2):24-33. https://doi.org/10.14198/cuid.2005.18.05.

9. Lopes L, Santos S. Florence Nightingale: apontamentos sobre a fundadora da Enfermagem Moderna. Rev. Enf. Ref. [Internet]. 2010; [citado 2020 ene 24];3(2):181-9. Disponible en: http://www.scielo.mec. pt/pdf/ref/vserllIn2/serllln2a19.pdf

10. Castro Molina FJ. La enfermera victoriana: género e imperio. Cultura de los Cuidados. 2016;46(3):42-51. http://dx.doi.org/10.14198/ cuid.2016.46.09.

11. Davies R. Notes on Nursing: what is and what is not (1860): by Florence Nightingale. Nurse Educ Today. 2012;32(6):624-6. http://dx.doi. org/10.1016/j.nedt.2012.04.025. PMid:22766202.

12. McDonald L. Florence Nightingale a Hundred Years on: who she was and what she was not. Womens Hist Rev. 2010;19(5):721-40. http:// dx.doi.org/10.1080/09612025.2010.509934. PMid:21344737.

13. Wells JS, Bergin M. British Icons and Catholic Perfidy - Anglo-Saxon historiography and the battle for crimean war nursing. Nurs Inq. 2016;23(1):42-51. http://dx.doi.org/10.1111/nin.12104. PMid:25982961.

14. Paredes M P, Rivas R E. Historia del Ejercicio Profesional de Enfermeras Hospitalarias del Sur de Chile. Cienc Enferm. 2014;20(1):9-21. http:// dx.doi.org/10.4067/S0717-95532014000100002.

15. Rivas E. Resumen de Ponencias VII Simposio Iberoamericano de la Historia de la Enfermería. $1^{a}$ ed. Temuco: Ediciones Universidad de La Frontera; 2019.

16. Carvalho C. Percepção, sensação e carnalidade na fenomenologia e ontologia de Maurice Merleau-Ponty. Argumentos. [Internet]. 2019; [citado 2020 ene 24];11(21):141-53. Disponible en: http://www.periodicos.ufc. br/argumentos/article/download/41050/97290/

17. Gadamer H-G. Verdad y método I. 5a ed. Salamanca: Sígueme; 1975. 
18. Grondin J. Hans-Georg Gadamer: una hermenéutica del acontecer de la comprensión. In Martínez A, editor. ¿Qué es la hermenéutica? Barcelona: Herder; 2006. p. 69-90.

19. Gadamer HG. Antología. Salamanca: Sígueme; 2001.

20. Austgard K. Doing it the gadamerian way - using philosophical hermeneutics as a methodological approach in nursing sciences. Scand J Caring Sci. 2012;26(4):829-34. http://dx.doi.org/10.1111/j.1471-6712.2012.00993.x. PMid:22515954.

21. Koskinen CA, Lindström U. Hermeneutic reading of classic texts. Scand J Caring Sci. 2013;27(3):757-64. http://dx.doi.org/10.1111/j.14716712.2012.01080.x. PMid:23004237.

22. Pérez A. Merleau-Ponty: percepción, corporalidad y cuerpo. Eikasia [Internet]. 2008; [citado 2019 nov 8];4(20):197-220. Disponible en: https://revistadefilosofia.org/20-06.pdf

23. Sartre J-P. Bosquejo de una Teoría de las Emociones. España: Alianza Editorial; 1987

24. Núñez Carrasco ER. Comprensión de la enfermería desde la perspectiva histórica de Florence Nightingale. Cienc Enferm. 2011;17(1):11-8. http:// dx.doi.org/10.4067/S0717-95532011000100002.

25. Pfettscher S. Florence Nightingale: la enfermería moderna. In Rodríguez M, editor. Modelos y teorías de enfermería. Greenville: Elsevier;2011. p.71-90.
26. Guimarães GL, Chianca TCM, Mendoza IYQ, Goveia VR, Matos SS, Viana LO. The core values of modern nursing in the light of Dilthey and Scheler. Texto Contexto Enferm. 2015;24(3):898-905. http://dx.doi. org/10.1590/0104-07072015003480014.

27. Fawcett $\mathrm{J}$. Analysis and evaluation of contemporary nursing: models and theories Philadephia. USA: Davis Company; 2000.

28. Valverde C. Enfermería centrada en los Significados del Paciente $®$ Un modelo basado en la narrativa y en la ética del otro. Index de Enfermería. [Internet]. 2008; [citado 2020 ene 24];17(3):1578. Disponible en: http://scielo.isciii.es/scielo.php?script=sci_ arttext\&pid=S1132-12962008000300001

29. Hernández Conesa JM. La historiografía del conocimiento: Florence Nightingale vs Ernesto Rusca. Temperamentvm. [Internet]. 2014 [citado 2019 nov 8];10(20):23-7. Disponible en: http://www.index-f. com/temperamentum/tn20/t2000.php

30. Sánchez M. El humanismo y la enseñanza de las humanidades médicas. Educ Med. 2017;18(3):212-8. http://dx.doi.org/10.1016/j. edumed.2017.03.001.

31. Goic A. El Sistema de Salud de Chile: una tarea pendiente. Rev Med Chil. 2015;143(6):774-86. http://dx.doi.org/10.4067/S003498872015000600011. PMid:26230561. 